\title{
PEER-TO-PEER CONTENT DISTRIBUTION An Analysis of the Internal and External Potentials and Obstacles
}

\author{
Jorn De Boever \\ Centre for Usability Research, K.U.Leuven, Parkstraat 45(b 3605), 3000 Leuven, Belgium \\ jorn.deboever@soc.kuleuven.be
}

Keywords: Peer-to-peer systems, content distribution, SWOT analysis, adoption.

\begin{abstract}
The popularity of (illegal) peer-to-peer file sharing has a disruptive impact on the internet traffic and the business models of content providers. In addition, several studies have found an increasing demand for bandwidth consuming content. It is widely recognised that illegal file sharing applications demonstrated the potential of peer-to-peer systems to distribute popular content in a scalable and inexpensive way. However, there has been relatively little economic analysis of the potentials and obstacles of peer-to-peer systems as a legal and commercial content distribution model. Many content providers encounter uncertainties regarding the adoption or rejection of peer-to-peer networks to spread content via the internet. The recent launch of several commercial, legal peer-to-peer content distribution platforms, which are still mainly beta versions, increases the importance of an integrated analysis of the internal strengths / weaknesses and the external opportunities / threats. This paper explores these internal and external aspects by means of an integrated SWOT analysis.
\end{abstract}

\section{INTRODUCTION}

Peer-to-peer systems have been associated with illegal file sharing since the introduction of Napster in 1999 (Shirky, 2001). But, this negative image has shifted because of the arrival of innovative peer-topeer systems that demonstrate the potential of these applications for legal content distribution. Nevertheless, we still notice that the adoption of peer-to-peer systems, to distribute content in a legal way, is still not widespread. In this context, the question arises which opportunities and challenges peer-to-peer systems offer for legal and commercial content distribution. Some technical aspects of peerto-peer networks - e.g. performance, information retrieval, and self organisation - have been extensively studied in recent years. However, there has been relatively little analysis of the economics of peer-to-peer systems. Consequently, we consider it to be important to have a broader more business oriented approach toward peer-to-peer platforms as a content distribution model.

The introduction of innovations causes a certain level of uncertainty for the industry, which must decide whether or not to adopt it (Rogers, 2003). The industry will try to compensate this uncertainty by exploring the different aspects of an innovation or by looking at what other companies have decided. That is why it is important to understand why companies would (not) adopt peer-to-peer systems and with which uncertainties the industry still struggles. We conducted an extensive SWOT analysis (Strengths, Weaknesses, Opportunities and Threats) by means of interviews and literature review. The aim of the present paper is therefore to briefly explore the obstacles and potentials of peerto-peer technology to distribute commercial content in an efficient and legal way. This study will allow us to evaluate the current business viability of peerto-peer technology. The results of this analysis should enable us to understand how peer-to-peer networks might be positioned strategically in order to raise the chances for a successful commercial application for legally exchanging media content. This paper comprises a study that might assist content providers in the adoption process.

This paper starts by reviewing some relevant literature in the area of economics and peer-to-peer systems, followed by the elaboration of the methodology. The next section presents the results of the SWOT analysis by enumerating these aspects. The final section will confront and link the internal and external elements so as to put meaning and interpretation to these results. 


\section{LITERATURE}

Substantial research effort has been devoted to the technical aspects of peer-to-peer technology. This has resulted in an extended body of literature about architectures (e.g. Androutsellis-Theotokis and Spinellis, 2004; De Boever, 2007), availability (e.g. Bhagwan, Savage and Voelker, 2003; Chu, Labonte and Levine, 2002), security (e.g. Divac-Krnic and Ackerman, 2005) and etc.

As we stated in the introduction, this paper will elaborate more on the economics of peer-to-peer technology. One of the most characteristic aspects of peer-to-peer systems is that - like in virtual communities - to a large extent, value is being created by the end users. End users are more than solely consumers in that they at least also cooperate to distribute the content (Lechner and Hummel, 2002). MacInnes and Hwang (2003) analysed four cases - KaZaA, Kontiki, SETI and Groove - of peer-to-peer business models. According to these authors, the most important challenges for the business models were the revenue model, security and user behaviour. In the study, conducted by Rupp and Estier (2003), different actors in the music industry were compared on how they were being confronted with and affected by new technologies such as peer-to-peer networks. Other studies explored the revenue models of several peer-to-peer systems (Hummel, Muhle and Schoder, 2005; Hummel, Strømme and La Salle, 2003). The revenue models of most existing file sharing applications failed on efficient allocation. This means that the revenues do not always end up with the rightful claimants. It is obvious that these studies already identified several of the issues that lead to uncertainty about the viability of peer-to-peer networks as content distribution models.

We will elaborate, integrate and build our findings on the studies that are stated below. The papers that will be integrated in our SWOT analysis are the following: Smith, Clippinger and Konsynski (2003), Hughes, Lang and Vragov (2005), Kwok, Lang and Tam (2002), Sigurdsson, Halldorsson and Hasslinger (2005) and Rodriguez, Tan and Gkantsidis (2006). The combinations of these studies with interviews provide us with a detailed analysis in which several new aspects have been identified. Although we value the results of the five selected studies, we argue that these papers suffer from some flaws which add to the significance of our contribution. For that reason, the drawbacks of these studies require further examination of the internal and external potentials and obstacles. We will now contend why these studies are deficient to a certain extent.

The study of Smith, Clippinger and Konsynski (2003) mainly reflects on the use of peer-to-peer technology within organizations (internally), whereas our analysis emphasizes peer-to-peer technology as a content distribution model in a commercial B2C (Business to Consumer) environment. Another limitation of their study is the fact that they only held discussions with CIOs, which results in only one point of view. In contrast, we have tried to recruit respondents with different backgrounds.

The research of Hughes, Lang and Vragov (2005) provides more of a theoretical, analytic framework for market design of peer-to-peer networks than it offers empirical results. We have integrated some elements of this framework in our study so as to corroborate our results. Another limitation of this study is that they primarily focus on the constraints - legal, technical, economic, structural, political, cognitive and socio-cultural while not paying attention to the strengths and opportunities.

Although Kwok, Lang and Tam (2002) use the words "risks and opportunities" in the title of their article, they limit their examination to the free riding problem, the related motivations for cooperation, and piracy. In addition, the analysis of these authors is merely from a theoretical perspective. Furthermore, these academics do not pay a lot of attention to the opportunities.

Sigurdsson, Halldorsson and Hasslinger (2005) provide an analysis that primarily focuses on technical and financial aspects while ignoring e.g. user-related aspects. Furthermore, we pose that these authors have failed to make the different concepts of a SWOT analysis operational, which resulted in confusion about the terms.

Finally, Rodriguez, Tan and Gkantsidis (2006) described the results of a workshop on the issues regarding legal peer-to-peer content distribution. This paper offers a list of items that stresses the weaknesses and threats, while paying little attention to the strengths and opportunities.

In this paper, we will integrate the results of these five studies, while addressing the flaws of former research in our own analysis.

\section{METHODOLOGY}

Our methodology consisted of three levels: a literature review, expert interviews and the data of 
the former two levels were used in a SWOT analysis. The first phase of our methodology comprised a detailed literature review. We already gave an overview of some of these articles above. From this literature review, we selected five papers to elaborate and integrate in our SWOT analysis. We only selected studies that covered a broad range of issues involved in peer-to-peer research and excluded others that only focus on one or a limited number of topics so as to make sure that overrepresentation of some topics would be avoided.

In the second phase, we conducted 15 in-depth interviews with people from the industry and with some academics. We have interviewed people with different backgrounds to obtain as accurate and as objective results as possible. Therefore, participants, with different backgrounds (social -3 , economic 3 , technical -8 , and law -1 ), were selected from different actors for these interviews. We interviewed respondents working for broadcasters (4), other content providers (4), ISPs (2), research community (4) and copyright lobby organisations (1). Of the researchers, two are working on research projects in which a peer-to-peer application is being developed. Based on the results of these interviews, we were able to accomplish a significant contribution to the existing literature by broadening the scope.

The findings of the former two phases resulted in the data for the SWOT analysis. First, we clearly defined what was meant by the concepts strengths, weaknesses, opportunities and threats so as to avoid confusion. Second, we scored the different topics that were mentioned in the results section of the papers and in the interviews for our analysis. Based on the defined concepts, we decided - by means of qualitative interpretation - for each item to which category it belonged. Third, based on literature review and the results of the in-depth interviews, we examined why the mentioned items were issues. Finally, we tried to confront or relate the items of the four categories by analysing e.g. which strengths might be used to address some weaknesses or to take advantage of certain opportunities; which weaknesses might be mended by further research; which threats must be avoided and etc.

Although SWOT analyses are mainly used within organisations (Vermeylen, 2004; Sabbaghi and Vaidyanathan, 2004), we argue that it is an effective tool as well to grasp the major positive and negative issues of applications and technologies. While studying technologies or information systems, researchers run the risk of considering the technology or the adoption of it as a positive fact. This has also been termed as the pro-innovation bias
(Rogers, 2003). The danger of the pro-innovation bias is that it becomes difficult to have a neutral and objective perspective. The advantage of a SWOT analysis is that the researcher is forced to analyse the weaknesses and threats as well. A SWOT analysis consists of two main parts, namely the internal aspects and the external aspects (Vermeylen, 2004). The internal aspects consist of the strengths and weaknesses. The external aspects contain the opportunities and threats. The external elements are the occurring changes, events, and etcetera that are taking place in the environment.

In the past and today, peer-to-peer networks have been very successful in sharing files, mostly in an illegal way. Peer-to-peer applications to share files or distribute content in a legal way are not widespread yet. The SWOT analysis will reveal several internal strengths / weaknesses and external opportunities / threats of peer-to-peer as a commercial application for the legal exchange of media content (video, images, text and film).

\section{RESULTS}

In this section, we will explore the internal strengths / weaknesses and external opportunities / threats. We will define what we understand under these concepts within each part of this section. The data in this section result from 15 interviews and five selected papers.

\subsection{Internal Strengths}

The strengths encompass the internal aspects that offer peer-to-peer systems for instance possibilities to take advantage of certain factors in the environment. These are the strengths that are characteristic of peer-to-peer systems.

Cost savings. Peer-to-peer is a technology that is capable of reducing the costs for content distribution because it utilizes fewer resources such as bandwidth, storage capacity and etc. (Norton, 2007; Smith, et al., 2003). In addition, the avoidance of centralised servers limits the costs in terms of investment and upkeep (Sigurdsson, et al., 2005). The lower costs make it possible for the industry to serve a larger audience or to distribute more bandwidth consuming content. This might lower the need for compression techniques so that the content has better quality. This makes peer-to-peer networks an interesting technology for the distribution of bandwidth consuming content. The implementation of techniques, such as swarming, makes it possible 
for end users to more efficiently download larger content. In other words, the industry does not have to buy that much distribution capacity anymore. It is also an inexpensive solution for the worldwide distribution of content. There is no need for adaptations on the network level, which reduces costs as well. All these aspects result from the fact that peer-to-peer systems utilise the available resources that are available for instance on the end users' personal computers. Norton (2007) has examined and compared several content distribution models based on a cost analysis. The result was that peer-to-peer systems were the most inexpensive content distribution models.

Scalability. Peer-to-peer systems scale with the amount of use, which results in limited marginal costs (Ding, Nutanong and Buyya, 2005; Taylor, 2004; Sigurdsson, et al., 2005). The extra costs for each additional user are restricted because each additional consumer in theory also means additional resources. These are positive network externalities of peer-to-peer systems which makes them very scalable. Some peer-to-peer networks are even able to cope with 'flash crowds' (i.e. a sudden and extremely increase of the number of users that consume the same service or content which might overload a system). Peer-to-peer technology might be utilised to develop overlay networks so as to distribute content around the globe. Furthermore, these overlay networks are cost-efficient as the costs for implementation and maintenance are limited (Sigurdsson, et al., 2005).

Easy implementation. From the position of content providers, peer-to-peer systems are easy and inexpensive to implement because it operates more on the application layer. To distribute content on a worldwide scale, there is little need for large investments in infrastructure and control. However, some peer-to-peer providers pose that the opposite is true. It is more complicated to start a peer-to-peer system in comparison with installing a central server.

Fault tolerance. Peer-to-peer systems are fault tolerant because there are limited centralised components in the network that are potential bottlenecks or single points of failure (Sigurdsson, et al., 2005). Because of the distributed nature of peerto-peer networks and the redundancy of nodes, the disappearance of one or several nodes mostly has only limited impact on the overall performance.

Availability. Some respondents consider peer-topeer systems to be more efficient because these systems utilise the idle resources that are available in the network. This leads to more availability of resources such as bandwidth, storage capacity, and content.

\subsection{Internal Weaknesses}

The internal weaknesses contain the distinctive characteristics of peer-to-peer networks that are disadvantageous. These are the direct constraints of these systems.

Quality of Service (QoS). The reliability of peerto-peer systems in terms of QoS is a major issue for the commercial viability of these systems (Parameswaran, Susarla and Whinston, 2001; Sigurdsson, et al., 2005; Hughes, et al., 2005; Rodriguez, et al., 2006). QoS in this context relates to the performance of the network for instance on the level of delays, content availability, distribution speed, scalability and etc. The end users' personal computers operate as nodes in the network. This makes it hard to guarantee service quality because these nodes can suddenly appear and disappear, and there are still end users with low bandwidth connections. Content might become unavailable if nodes, that host certain content, suddenly leave the system. This might result in delays and high buffer times. This is especially problematic for large content that takes more time to download. If the download requires more time, than there is more chance that the content exchange cannot be completed because the hosts become unavailable. The more decentralised a peer-to-peer network is, the more that QoS is an issue. The service quality might also influence the real time experience of peer-to-peer live streaming applications. Peer-topeer content distribution is in this sense a best effort model with low guarantees of service quality. The uncertain QoS is probably the most important obstacle for content providers to adopt peer-to-peer systems for content distribution. In addition, it remains unclear whether users will accept that content, from professional content providers, that is being shared in a legal way, is unavailable or requires delays. In summary, it seems difficult to guarantee a certain performance level and it is therefore hard to formulate reliable service level agreements.

Inefficient use of resources. Most peer-to-peer systems handle the available resources - e.g. bandwidth - in an inefficient way. This results from the fact that there are mostly no centralised components in the system that manages the use of these resources because peer-to-peer networks are, to a large extent, self organising systems. This self organisation requires much traffic to maintain the 
operability of the system. This was e.g. a significant issue in a former Gnutella version (e.g. Ripeanu, Iamnitchi and Foster, 2002).

Security and privacy. These issues are still worrisome within the context of peer-to-peer systems because of decentralization (Taylor, 2004; Smith, et al., 2003). Peer-to-peer implies that other peers can access your computer to retrieve certain data. This makes it easy for malicious nodes to enter your computer and cause damage. This danger for malicious attacks has social implications as well, related to trust (Rodriguez, et al., 2006). In most peer-to-peer systems, users encounter unknown anonymous peers they should trust if they want to exchange data. Inflicting damage in this trust relation might cause users to abandon peer-to-peer systems. The 'older' generations of peer-to-peer systems are mainly anonymous without actual social interaction, which limits the trust relation among peers. In addition, the lack of centralised monitoring facilities causes difficulties in guaranteeing security (Sigurdsson, et al., 2005). By using peer-to-peer networks, users expose private data such as IP address, the content they have consumed and etc. This can inflict damage to the users' privacy (Rodriguez, et al., 2006).

Usability and adoption. These are two issues that received little attention in peer-to-peer research and it proved to be a concern for some of the respondents. Most peer-to-peer file sharing applications have complex interfaces that are little intuitive. Several terms (e.g. seeders, trackers and etc.) might have no meaning to some users. Good and Krekelberg (2005) have conducted usability research on the KaZaA user interface. They came to the disquieting result that many users were not able to locate their shared folder, so they did not know which content they were actually sharing. This is probably the reason why these researchers found personal data on KaZaA such as e-mails and even credit card data. Today, peer-to-peer systems are mainly used by persons who regularly surf the internet and who probably know how peer-to-peer networks function. If one wants to develop peer-topeer applications to be consumed by a wide audience, the user interface needs to be more accessible, usable and learnable for users who are less familiar with peer-to-peer systems (e.g. Morris, 2007). For instance, the fact that users have to download and install a separate client might be a threshold to adopt these systems. Furthermore, file sharing might frustrate users as they have to wait until the full file has been downloaded before consuming it (Rodriguez, et al., 2006).
Costs to users. Peer-to-peer systems utilise resources that are available on the end users' personal computers. The users' resources - e.g. storage capacity, bandwidth, electricity - are being used for the distribution of content, which entails considerable costs for these users. It remains an open question whether the average user will accept that a content provider utilises the user's resources to distribute content.

Business model. Peer-to-peer systems are being confronted with indistinctness's and strains regarding several elements of business models. There is still no proof of concept of a clear, comprehensive business model that integrates peerto-peer systems and where there is a fit between the several business model components. The main bottlenecks in this area, according to most respondents, are related to control. The lack of control over large parts of content distribution is problematic to some content providers. In addition, there are often no means to measure the consumption of the peers, which makes it difficult to attract advertisers. There exists no proof of concept of peer-to-peer systems that integrate an advertising or payment model for revenues. Peer-to-peer systems therefore need a centralised component which monitors the consumption of the peers in order to be able to implement a revenue model.

\subsection{External Opportunities}

The opportunities contain the external elements to which peer-to-peer systems can anticipate from its internal assets. In other words, these topics consist of the aspects in the environment that might have a positive effect on the adoption and use of peer-topeer systems. The opportunities of peer-to-peer networks often result from the flaws of other distribution models.

Costs centralized models. In a client/server network where there is a situation of augmenting popularity of bandwidth consuming content - such as audio, video and games - the costs for distributing this content will increase. Furthermore, the risk of a bottleneck and single point of failure increases as well. Researchers have noticed an increasing use of and demand for bandwidth consuming content such as video, audio, HDTV and etc. (IDC, 2007). These researchers predict that the amount of digital data will expand even more in the following years. Some experts predict that video content will have a share of about $80 \%$ of the internet traffic (Norton, 2007). For centralised distribution models, this implies additional costs in 
the sphere of for instance distribution capacity, storage capacity, bandwidth, servers, control and etc.

Disadvantages of other distribution models. We will demonstrate some opportunities for peer-to-peer systems by addressing some flaws of other distribution models such as unicasting, multicasting and CDNs (Content Delivery Network). Unicasting is a centralised streaming method that has the same drawbacks as client/server models. Unicasting does not scale with the amount of consumption, it runs the risk of a bottleneck and the costs rise as content is consumed more often. Therefore, most content providers that utilise a unicasting model, limit the quality of the content to confine the costs when popularity increases. Multicasting is a more decentralized streaming method, but this technique has some drawbacks as well. Multicasting requires adaptations in the network infrastructure and many networks are not multicast enabled. Therefore, multicasting is not suitable for worldwide content distribution. Furthermore, multicasting is mainly interesting for live content distribution and less for on demand content. A third example of a distribution model is a CDN, which is a distributed network of computers to distribute content. An elaborated analysis of CDNs can be found in the text of Pallis and Vakali (2006). When content providers decide to utilise a CDN, they first make strategic decisions about the necessary performance and QoS and include these requirements in a service level agreement. But, it is often difficult to predict the popularity of services or content which might influence the service quality.

Increasing capacity of end users' equipment. The end users possess an expanding amount of resources - such as bandwidth, storage and processing capacity - that can be exploited by peer-to-peer networks (Sigurdsson, et al., 2005). This also means that there are more idle resources available on the edge of the internet. Peer-to-peer systems have become a success because of the availability of unutilised resources. Users have become more than purely consumers in the value chain and they now add value by replicating and distributing content in peer-to-peer systems.

Evolution toward symmetric networks. The internet today is still organised in an asymmetric way, i.e. it expects users more to download and less to upload content. This is why users have more download capacity in comparison with their upload capacity. But this trend is shifting because of developments such as peer-to-peer file sharing networks and, more recently, user generated content. Several respondents expected the internet to become more symmetrically organised in the future so that the down-/upload ratio will gradually equals one. This might be an opportunity for systems such as peer-to-peer networks that require users to utilise their upload capacity to serve content.

User orientation. Several respondents posed that peer-to-peer networks focus more on the end users by giving them more roles in the value chain. In peer-to-peer file sharing systems, a social filtering process occurs in which the community of peers decide which content might remain available and which content not. Content that does not fit the users' needs, or that becomes less relevant, will slowly disappear from the system. In other words, peer-to-peer systems offer the opportunity to involve the end users more closely in the value chain.

\subsection{External Threats}

The external threats are factors in the external environment that can interrupt or threaten the success of commercial and legal peer-to-peer systems.

Data limits. ISPs or network providers still maintain data limits in several countries as a part of their business model and to be able to guarantee a certain level of performance and control over the traffic. If a content provider wants to distribute large content such as video files, end users will soon attain their data limit. This is problematic since more content providers want to distribute video over the internet in high definition. If users exceed the allowed limit, they need to pay for the extra consumed volume.

Asymmetric networks. The commercial internet has been build from a top down perspective. The content owners distribute content, and the users download this content. In other words it focuses more on the download link, which resulted in asymmetric organisation, e.g. ADSL. This asymmetric architecture causes low upstream rates (Sigurdsson, et al., 2005). For the efficient operation of peer-to-peer systems, it would have been better to have a more symmetrical relationship between upand downloading - for instance SDSL - because peer-to-peer systems emphasise uploading as well. In the current asymmetric organisation of the internet, there is a risk that the upstream bandwidth capacity will not be sufficient to compensate the downstream bandwidth capacity in a situation of widespread peer-to-peer traffic (Norton, 2007). If the use of peer-to-peer systems would be ubiquitous, the ISPs fear that the upload capacity might become congested during peak hours. 
Measurability and control. In server oriented models, it is easier to keep track of the different flows of data in the system which is a prerequisite for advertisers and content owners (Smith, et al., 2003; Sigurdsson, et al., 2005). This measurability is important to the revenue model within the business model. This measurability of other distribution models is a threat to peer-to-peer networks and it is an internal weakness as well.

Competing distribution models. By means of some examples, namely multicasting and CDNs, we will demonstrate that peer-to-peer systems are being threatened by some characteristics that other models display. In the assessment of using a certain content distribution model, content providers will balance the pros and cons of these systems against each other in order to make the right decision. Multicasting, for instance, is a streaming model that offers some answers to the flaws of the unicasting model, particularly in the area of live streaming. In addition, multicasting is a system that reinforces itself so that it is very scalable. Furthermore, multicasting can offer better QoS compared to peer-to-peer streaming. In this sense, multicasting can be considered as a more reliable model that is mainly suitable for popular live events. A second example of content distribution models contains CDNs. In contrast with peer-to-peer systems, CDNs offer high guarantees on the level of QoS which makes it possible to draw up reliable service level agreements. In general, CDNs are considered to be reliable content distribution models that are able to address several security issues.

Position of ISPs. We will now explain why ISPs might not sympathize with peer-to-peer content distribution models (Rodriguez, et al., 2006). Content providers, that implement peer-to-peer technology for content distribution, avoid bandwidth expenses by passing the costs on the users. The users will try to prevent these costs by making sure that they do not exceed their data limit. The result of the adoption of peer-to-peer technology is an increasing traffic volume on the network infrastructure of ISPs, while these ISPs receive less revenues. Measurements studies have demonstrated that more than $50 \%$ of internet traffic is emanating from peerto-peer systems (Haßlinger, 2005; CacheLogic, 2004). The increasing traffic volume (especially the upstream traffic), caused by peer-to-peer systems, originates from the fact that peer-to-peer technology allows exchanging large files and because there is much message overhead required to self organise the system. In addition, most peer-to-peer applications lack locality awareness which implies that a lot of traffic crosses several ISP networks whereas the content might be available on a node of the local ISP (Rodriguez, et al., 2006). In other words, the current revenue model of ISPs does not fit a situation in which the use of peer-to-peer networks is pervasive because the traffic volume might not be in proportion to the revenues. If large traffic volumes are congesting the network, ISPs might decide to squeeze, queue or even block peer-to-peer traffic. We have to remark that ISPs will not be likely to go that far because these actions can cause unfavourable churn rates. The consequence might be that many ISPs change their price policy from e.g. flat rates into a system of layered pricing according to the traffic users generate (Rodriguez, et al., 2006).

Legal issues and image. Peer-to-peer technology has to cope with an image problem, in which there is still a connotation of illegal file sharing, piracy and sharing of pornographic content. This is why some authors (e.g. Rodriguez, et al., 2006) argue that legal uses of peer-to-peer content distribution should be promoted. The MP3 encoding format, combined with the exchange possibilities of peer-to-peer file sharing, have lead to a situation in which illegal file sharing has become an omnipresent and accessible practice that is not likely going to disappear (Kwok, et al., 2002; Smith, et al., 2003; Sigurdsson, et al., 2005). Decentralisation makes it difficult to monitor and manage peer-to-peer networks. Making content downloadable is difficult from a business perspective, because content owners fear copyright infringements. If users are able to store content on their hard drives, the chances increase that files are being copied and spread in an illegal way. Control over the rights remains an important issue. However, several respondents posed that control of copyrights was not their major concern. They emphasised the importance of control of their business model. These respondents accept for instance that content is being copied and distributed by the end users, as long as they are able to capture these 'eyeballs' for advertising purposes. On the other hand, most of the interviewees hold an opinion that solutions such as DRM are not suitable for fighting piracy because (1) every version will be compromised sooner or later and (2) it does not fit the consumers' needs. In addition peer-to-peer providers have to cope with different legislations in different countries. For instance, the USA has developed the fair use doctrine, whereas European jurisdiction utilises an exception list. For a more in-depth analysis of jurisdiction concerning illegal file sharing in different countries, we refer to (Werkers and Gilio, 2006; Dogan, 2005). 
Critical mass. The operation and viability of peer-to-peer systems require a critical mass of cooperating users that make sufficient resources available (Rodriguez, et al., 2006; Kwok, et al., 2002). In client/server systems, it does not matter that content is consumed by little people as long as the server stays available. If a new peer-to-peer application is being introduced, it must generate sufficient mass so that content would be sufficiently available. But, as is the case in many virtual communities, peer-to-peer networks are being threatened by the free riding phenomenon (Adar and Huberman, 2000; Hughes, Coulson and Walkerdine, 2005). Free riders are users that consume resources from the network while not contributing anything in return. In a situation without critical mass and a large amount of free riders, content will often be unavailable. This causes users to leave the system with the result that the peer-to-peer network is no longer viable. Consequently, peer-to-peer technology is mainly interesting for the distribution of popular content because it is easier to attain a critical mass of cooperating peers.

Content aggregation. Several peer-to-peer platforms - such as Babelgum and Joost - operate as content aggregators as well. Peer-to-peer platforms try to acquire popular content to increase the popularity of their service. A part of the revenue model of several peer-to-peer platforms is based on advertising and revenue sharing (e.g. Warner Music Group, 09.04.2007). There are several competing peer-to-peer companies trying to gather the same content providers. As more peer-to-peer systems will start up, it will become more difficult to aggregate popular content providers and as a result to attain a critical mass. This threat of content aggregation is linked to the issues of competing distribution models and the acquisition of a critical mass.

Decreasing bandwidth prices, Network Address Translation (NAT) and abandonment of open source. The first issue concerns the relevance of peer-to-peer content distribution if the bandwidth prices would decrease significantly. Peer-to-peer content distribution is mainly regarded as a technology to save on bandwidth costs. As bandwidth capacity might become cheaper, the question arises whether a content provider would still adopt a distribution model that performs badly in terms of service quality. The second issue, NATs, involves mainly a technical burden that needs to be solved (Rodriguez, et al., 2006). The use of a NAT makes that several hosts within a private network only use one IP address to have access to the internet. The use of NATs is increasing for instance because of the use of wireless routers. The exchange with nodes that operate behind a NAT router generates a technical issue, by limiting end-to-end connectivity, which needs to be addressed. The final issue relates to the abandonment of open source because of commercialisation of peer-to-peer technology. Most commercial peer-to-peer providers use closed source. Closed source software is not subject to peer review with the result e.g. that it might be engineered poorly and that it is less reliable.

\section{CONFRONTATION}

Some people criticise SWOT analyses for being just an enumeration of several points without any reflection (Vermeylen, 2004). We therefore will try to discover links between the internal and external aspects that have an impact on peer-to-peer systems. Peer-to-peer systems can take advantage of some external opportunities by deploying the internal strengths. Research and experiments might be necessary to address the internal weaknesses, while peer-to-peer providers are sometimes not able to change the external threats.

The results of the SWOT analysis indicate that peer-to-peer networks still have not been able to break through their infancy. The identified limitations of peer-to-peer technology still entail major issues on the way to a legal and commercial content distribution model. The relation between the strengths and opportunities is certainly existent. Particularly, peer-to-peer networks can be perceived as inexpensive, fault tolerant and scalable content distribution models. These internal assets can be used to address some external opportunities. Peer-topeer technology provides solutions for some of the flaws of other content distribution models, such as the client/server model (e.g. bottleneck, not scalable), unicasting (e.g. bottleneck, not scalable), multicasting (e.g. network adaptations). The costs of several current distribution models will increase as demand for popular bandwidth consuming content grows. Peer-to-peer technology distributes content in a cost efficient manner that requires less limiting the quality of the content by compression techniques. In addition, the possible evolution toward symmetric networks might create opportunities for peer-to-peer systems to utilise the users' uplink even more.

On the one hand, there are several weaknesses for instance on the level of usability and business modelling - that still can be mended. These are elements that require further research and experimentation, but these are not insurmountable 
problems. On the other hand, other weaknesses (e.g. QoS and security) and threats (e.g. legal issues, control) require the integration of a centralised component in the peer-to-peer architecture. Moreover, as we indicated, a business model requires that companies are able to monitor and measure the consumption of users, to support for instance the advertising model. To pursue these goals, a centralised component is necessary in the architecture. Finally, to improve the QoS of peer-topeer systems, it might be an option to close agreements with ISPs to equip its infrastructure so that the traffic becomes more manageable. In this way, ISPs might be able to generate revenue from the additional load on their infrastructure, caused by traffic of peer-to-peer networks. For instance, BitTorrent has closed a service level agreement with GNi, a company that offers data networking solutions, so as to be able to offer better user experience (Del Conte, 28.06.2006).

Most of the external threats and opportunities are beyond the manipulation of peer-to-peer providers. Peer-to-peer providers can only take advantage of the opportunities by promoting the criticalities of these aspects (e.g. demand for bandwidth consuming content, costs of centralised models and etc.). On the level of threats, a strategy might include avoiding these elements, although this seems to be impossible. Peer-to-peer providers cannot ignore issues such as data limits, asymmetric networks and the control of ISPs. Every peer-to-peer provider, that offers solutions for the distribution of popular bandwidth consuming content, is being confronted with these threats.

We will now describe a worst case scenario, in which peer-to-peer content distribution is massively used for legal content distribution and in which the threats have not been addressed. In a situation in which peer-to-peer systems would be used by the average internet user, and not only by young more computer literate users, the positive network externalities of peer-to-peer networks might fall into negative network externalities. The network infrastructure of the ISPs would be overloaded which would result in insufficient QoS, which would reveal itself in for instance delays. To retain control of the traffic, ISPs might decide to squeeze or to block peer-to-peer traffic which would decrease user experiences. Because of these inadequate user experiences, users might decide to stop using the peer-to-peer system with the result that content would be less replicated throughout the network. The unstable availability of content will cause additional loss of users, which results again in less available resources. In other words, this vicious circle would prevent peer-to-peer systems to attain a critical mass resulting in the extinction of peer-topeer systems. This is a worse case scenario that is not likely going to occur because peer-to-peer providers will try to avoid or address these weaknesses and threats.

\section{CONCLUDING REMARKS}

The present study examined the internal/external potentials and obstacles of peer-to-peer systems to distribute commercial content in an efficient and legal way. Data gathered from interviews and literature was utilised to conduct an extensive SWOT analysis from a business perspective. Overall, this study reflected previous research findings (Smith et al., 2003; Hughes et al., 2005; Kwok et al., 2002; Sigurdsson et al., 2005 and Rodriguez et al., 2006). We argue that our research succeeded in addressing the limitations of former research as was discussed in the literature section. Interestingly, by conducting interviews with several respondents with different backgrounds, this SWOT analysis added several new elements to the existing results in a structured way. Whereas most researchers primarily examined the internal weaknesses, this study highlighted the importance of the internal strengths and external opportunities/threats as well. In addition, this examination paid important attention to the position of the users, since the users are essential in the value creation within the peer-to-peer value chain. On the one hand, users have more resources - e.g. bandwidth or storage capacity - at their disposal, which creates opportunities for peer-to-peer systems. On the other hand, the use of peer-to-peer systems implies that users experience additional costs because they have to make these resources available for other peers in the system. Furthermore, usability requirements of peer-to-peer systems have received little attention. This lack of knowledge might impede the widespread adoption of peer-to-peer systems among end users. Finally, a large proportion of users, in a great deal of peer-to-peer systems, are free riding on the contributions of others. There is a need for incentives to increase cooperation so as to ensure network performance.

Beside users, we focussed on another often forgotten actor as well, namely the ISPs. Peer-topeer systems cause an increased load on the network infrastructure of ISPs, while the ISPs are less remunerated. This causes a negative attitude of ISPs 
toward peer-to-peer systems, which might motivate the ISPs to hinder peer-to-peer traffic.

In comparison with the five selected papers, our research added the following topics: strengths (easy implementation, availability), weaknesses (usability and adoption, costs to users, business models), opportunities (disadvantages of other distribution models, evolution toward symmetric networks, user orientation) and threats (data limits, competing distribution models, content aggregation, decreasing bandwidth prices, abandonment open source).

In contrast with former studies, this examination goes beyond a mere enumeration of aspects by confronting and linking the internal and external elements. Several external developments offer opportunities for peer-to-peer technology. In this sense, peer-to-peer content distribution can be envisaged as an inexpensive, fault tolerant and scalable model for content distribution that offers some advantages in comparison with other content distribution models. Some of the internal weaknesses can be addressed by further examination, for instance on the level of usability and business modelling. The QoS, peer-to-peer systems produce, remains the major obstacle for a widespread adoption of peer-to-peer technology among content providers. Most of the external threats and opportunities are beyond the control of peer-to-peer providers. Peer-to-peer providers cannot avoid issues - such as asymmetric networks, data limits - which threatens their commercial position.

Despite of the above mentioned positive qualities of this analysis, we have to address the limitations of this study as well. The reliability of this examination might be threatened by the underrepresentation of some actors in the interviews. We only conducted a limited amount of interviews with copyright lobbyists (1) and ISPs (2). However, we did not manage in recruiting peer-to-peer providers for this analysis which might have affected the results. In this sense our results might primarily represent the issues identified by content providers. In addition, many issues have been raised involving users whereas this research did not gather data from end users. Indeed, a promising line of study would be to interview peer-to-peer providers and end users so as to improve the reliability of the results.

Overall, the results of this study imply that peerto-peer technology offers some opportunities for commercial and legal content distribution. The recent launch of several legal, commercial peer-topeer systems - e.g. Zattoo, Babelgum, Joost, Vuze have demonstrated the feasibility of these models.
However, the results of this study indicate that several issues still need to be addressed. Centralising some elements of the architecture might be necessary to address the weaknesses - e.g. security, QoS - of this content distribution model. In conclusion, we pose that further development and examination of peer-to-peer technology is still a prerequisite for commercial viability.

\section{ACKNOWLEDGEMENTS}

FLEET (an interdisciplinary research project on FLEmish E-publishing Trends) is an IWT SBO project, with research partners IBBT SMIT, Cemeso, LSTS, MOFI, ICRI, CUO, MICT, ECDC and TNO.

\section{REFERENCES}

Adar, E. \& Huberman, B.A. (2000). Free Riding on Gnutella. First Monday, 5(10), Retrieved March 8, 2006, from http://www.firstmonday.dk/issues/issue5_10/adar/.

Androutsellis-Theotokis, S. \& Spinellis, D. (2004). A Survey of Peer-to-Peer Content Distribution Technologies. ACM Computing Surveys, 36(4), 335371.

Bhagwan, R., Savage, S. \& Voelker, G.M. (2003). Understanding Availability. Paper presented at the $2^{\text {nd }}$ International Workshop on Peer-to-Peer Systems, Berkeley, USA.

CacheLogic (2004). The True Picture of Peer-to-Peer Filesharing. CacheLogic, Retrieved November 23, 2006,

from http://www.cachelogic.com/home/pages/research/p2p2 004.php.

Chu, J., Labonte, K. \& Levine, B.N. (2002). Availability and Locality Measurements of Peer-to-Peer File Systems. Paper presented at the Conference on Scalability and Traffic Control in IP Networks, Boston, USA.

De Boever, J. (2007). Peer-to-Peer Networks as a Distribution and Publishing Model. Proceedings of the $11^{\text {th }}$ International Conference on Electronic Publishing, Vienna, Austria, 175-188.

Del Conte, N.T. (28.06.2006). BitTorrent pays for direct delivery. PC Magazine, Retrieved September 26, 2007, from http://www.pcmag.com/article2/0,1895,1983053,00.as p.

Ding, C.H., Nutanong, S. \& Buyya, R. (2005). Peer-toPeer Networks for Content Sharing. In R. Subramanian \& B.D. Goodman (Eds.). Peer-to-Peer Computing: the Evolution of a Disruptive Technology (pp. 28-65). London: Idea Group Publishing. 
Divac-Krnic, L. \& Ackerman, R. (2005). Security-Related Issues in Peer-to-Peer Networks. In R. Steinmetz \& K. Wehrle (Eds.). Peer-to-Peer Systems and Applications (pp. 529-545). Berlin Heidelberg: Springer-Verlag.

Dogan, S.L. (2005). Peer-to-Peer Technology and the Copyright Crossroads. In R. Subramanian \& B.D. Goodman (Eds.). Peer-to-Peer Computing: the Evolution of a Disruptive Technology (pp. 166-193). London: Idea Group Publishing.

Good, N.S. \& Krekelberg, A. (2005). Usability and privacy: a study of KaZaA P2P file-sharing. In L.F. Cranor \& S. Garfinkel (Eds.). Security and Usability: Designing Secure Systems That People Can Use (pp 651-668). Cambridge: O’Reilly.

Haßlinger, G. (2005). ISP Platforms Under a Heavy Peerto-Peer Workload. In R. Steinmetz \& K. Wehrle (Eds.). Peer-to-Peer Systems and Applications (pp. 369-381). Berlin Heidelberg: Springer-Verlag.

Hughes, D., Coulson, G. \& Walkerdine, J. (2005). Free Riding on Gnutella. IEEE Distributed Systems Online, 6(6).

Hughes, J., Lang, K.R. \& Vragov, R. (2005). Electronic Market Design Principles in the Context of Peer-toPeer Filesharing Systems. Paper presented at the Pacific Asia Conference on Information Systems, Bangkok, Thailand.

Hummel, T., Muhle, S. \& Schoder, D. (2005). Business Applications and Revenue Models. In R. Steinmetz \& K. Wehrle (Eds.). Peer-to-Peer Systems and Applications (pp. 473-489). Berlin Heidelberg: Springer-Verlag.

Hummel, T., Strømme, Ø. \& La Salle, R.M. (2003). Earning a Living among Peers - the Quest for Viable P2P Revenue Models. Paper presented at the $36^{\text {th }}$ Hawaii International Conference on System Sciences, Island of Hawaii (Big Island), Hawaii.

IDC (2007). The Expanding Digital Universe: A Forecast of Worldwide Information Growth Through 2010. EMC. Retrieved April 4, 2007, from http://www.emc.com/about/destination/digital_univers e/pdf/Expanding_Digital_Universe_Executive_Summ ary_022507.pdf.

Kwok, S.H., Lang, R.L. \& Tam, K.Y. (2002). Peer-to-Peer Technology Business and Service Models: Risks and Opportunities. Electronic Markets, 12(3), 175-183.

Lechner, U. \& Hummel, J. (2002). Business Models and System Architectures of Virtual Communities: From a Sociological Phenomenon to Peer-to-Peer Architectures. International Journal of Electronic Commerce, 6(3), 41-53.

MacInnes, I. \& Hwang, J. (2003). Business Models for Peer to Peer Initiatives. Paper presented at the $16^{\text {th }}$ Bled Electronic Commerce Conference, Bled, Slovenia.

Morris, I. (2007). Crave Talk: Five things that are wrong with BBC iPlayer. CNET. Retrieved July 31, 2007, from

http://crave.cnet.co.uk/software/0,39029471,49291924 ,00.htm.
Norton, W.B. (2007). Video Internet: The Next Wave of Massive Disruption to the U.S. Peering Ecosystem. Paper presented at the Stanford Networking Seminar, Stanford, USA.

Pallis, G. \& Vakali, A. (2006). Insight and Perspectives for Content Delivery Networks. Communications of the ACM, 49(1), 101-106.

Parameswaran, M., Susarla, A. \& Whinston, A.B. (2001). P2P Networking: An Information-Sharing Alternative. IEEE Distributed Systems Online, 34(7), 1-8.

Ripeanu, M., Iamnitchi, A. \& Foster, I. (2002). Mapping the Gnutella Network. IEEE Internet Computing, 6(1), 50-57.

Rodriguez, P., Tan, S.-M. \& Gkantsidis, C. (2006). On the Feasability of Commercial, Legal P2P Content Distribution. ACM SIGCOMM Computer Communication Review, 36(1), 75-78.

Rogers, E.M. (2003). Diffusion of Innovations. New York: Free Press.

Rupp, P. \& Estier, T. (2003). A Model for a Better Understanding of the Digital Distribution of Music in a Peer-to-Peer Environment. Paper presented at the $36^{\text {th }}$ Hawaii International Conference on System Sciences, Island of Hawaii, (Big Island), Hawaii.

Sabbaghi, A. \& Vaidyanathan, G. (2004). SWOT Analysis and Theory of Constraint in Information Technology Projects. Information Systems Education Journal, 2(23), 3-19.

Shirky, C. (2001). Listening to Napster. In A. Oram (Ed.), Peer-to-Peer: Harnessing the Power of Disruptive Technologies (pp. 21-37). United States of America: O’Reilly \& Associates, Inc.

Sigurdsson, H. M., Halldorsson, U.R. \& Hasslinger, G. (2005). Potentials and Challenges of Peer-to-Peer Based Content Distribution. Paper presented at the $2^{\text {nd }}$ International CICT Conference: Next Generation Broadband - Content and User Perspectives, Lyngby, Denmark.

Smith, H.A., Clippinger, J. \& Konsynski, B. (2003). Riding the Wave: Discovering the Value of P2P Technologies. Communications of the Association for Information Systems, 11(4), 94-107.

Taylor, I.J. (2004). From P2P to Web Services and Grids: Peers in a Client/Server World. London: Springer.

Vermeylen, S. (2004). Werken met SWOT-analyse. Brussel: Uitgeverij Politeia.

Warner Music Group (09.04.2007). Warner Music Group and Joost announce agreement to offer WMG video content on new internet television platform. WMG. [26.09.2007,

WMG: http://www.wmg.com/news/article/?id=8a0af81211b8 cea50111d683c13e0f66].

Werkers, E. \& Gilio, F. (2006). De complexe verhouding tussen peer-to-peer netwerken en de exceptie van de privékopie: kan de driestappentest een evenwicht tot stand brengen? Computerrecht, 166, pp. 283-292. 